\title{
Spectroscopic analysis of amyloid fibril formation in SH3-domains
}

\author{
Salvador Ventura* and Luis Serrano \\ European Molecular Biology Laboratory, Meyerhofstrasse 1, D-69117 Heidelberg, Germany
}

\begin{abstract}
The aggregation of proteins in fibrillar form is a problem of critical importance in a wide range of abnormal disease states. To decipher the molecular mechanism of formation of protein fibrillar aggregates we have chosen to study as model SH3 domains that exhibit different abilities to polymerize into amyloid fibrils. While being not related to any known disease, the SH3 domain of the p85 $\alpha$ subunit of phosphatidylinositol 3 kinase has been found to form amyloid fibrils in vitro under acidic conditions, meanwhile, we have found that the spectrin SH3-domain, sharing the same fold and some sequential identity keeps its native conformation under the same conditions. The use of spectroscopic methods to study these properties is illustrated in the present job, and correlated to direct sample observation by electron microscopy.
\end{abstract}

Keywords: Amyloid formation, UV, fluorescence, circular dichroism, SH3 domain

\section{Introduction}

Amyloidoses are a group of protein misfolding diseases that are characterised by the polymerisation of normally innocuous and soluble proteins or peptides into insoluble proteinaceous deposits. Among these diseases, we can point out the transmissible spongiform encephalopathies, Alzheimer's disease and type II diabetes [1]. The proteins responsible for these diseases do not share structural or sequential identities [2]. In spite of this diversity, all amyloid fibrils display similar features that can be checked by different spectroscopic methods: (1) they are long, straight and unbranched fibrils with a diameter between 40-120 ; (2) they bind to dyes such Congo Red and thioflavin-T, and (3) X-ray fibril diffraction and circular dichroism (CD) studies have indicated that they all exhibit a cross- $\beta$ structure. In the last few years, proteins unrelated to any known human disease have been found to convert in vitro into higher order structures that also present a cross- $\beta$ conformation and fulfill all spectroscopic characteristics of amyloid fibrils [3-5]. This has suggested that the ability to form amyloids might be a general property of proteins. One way to test for this hypothesis is to analyse proteins evolutionary related with similar structures and low sequence homology. We choose two different SH3 domains. SH3 domains are all $\beta$-sheet proteins domains of around 60 aa involved in protein-protein interaction [6]. The SH3 domain of the p85 $\alpha$ subunit of phosphatidylinositol 3 kinase has been found to form amyloid fibrils in vitro under acidic conditions [4]. In contrast to PI3-SH3, we have found, that the related $\alpha$-spectrin-SH3 (SPCSH3), which has the same fold and certain sequence identity with PI3-SH3, does not form amyloid fibrils under the same conditions [7]. By protein engineering methods we have also studied structural/sequential features responsible for their different ability to polimerize into fibrils [7]. Here we illustrate in more detail the different spectroscopic techniques used to explore such differential behaviour.

${ }^{*}$ Corresponding author. Tel.: + 349358121 54; Fax: + 349358112 64; E-mail: salvador.ventura@uab.es. 


\section{Materials and methods}

\subsection{Fibril preparation}

Native protein samples were prepared in buffer $\mathrm{N}$ (50 mM sodium phosphate $\mathrm{pH} 7.0)$. Samples at $\mathrm{pH}$ 2.0 were obtained either by a $\mathrm{pH}$ jump from $\mathrm{pH} 7.0$ or by dyalisis against buffer $\mathrm{F}$ ( $20 \mathrm{mM}$ glycine buffer). Fibril samples were obtained by incubating protein at $10 \mathrm{mg} / \mathrm{ml}$ in buffer $\mathrm{F}$ at room temperature or at $4{ }^{\circ} \mathrm{C}$ for several hours to several days.

\subsection{Circular dichroism}

Circular dichroism (CD) spectra in the far-UV region was obtained by using a jasco 710 spectropolarimeter at $20^{\circ} \mathrm{C}$. Spectra were recorded for native proteins $(10 \mu \mathrm{M})$ at $\mathrm{pH} 7.0$ and for proteins after incubation at $\mathrm{pH} 2.0$ for several days. Twenty accumulations were averaged to obtain each spectrum.

\subsection{Thioflavin-T binding and staining assays}

Samples $(5 \mu \mathrm{l})$ at $10 \mathrm{mg} / \mathrm{ml}$ in buffer $\mathrm{F}$ were diluted in reaction buffer $(10 \mathrm{mM}$ sodium phosphate/ $150 \mathrm{mM} \mathrm{NaCl})$ containing $65 \mu \mathrm{M}$ thioflavin-T $(1 \mathrm{ml})$. Samples were taken into and out of the pippete to facilitate the disruption of large aggregates and fluorescence data were collected after 5 min to ensure thermal equilibrium. Fluorescence excitation spectrum was recorded with emission intensity collected at $482 \mathrm{~nm}$. For thioflavin-T staining, aliquots of protein at $10 \mathrm{mg} / \mathrm{ml}$ in buffer $\mathrm{F}$ were air dried and stained with $0.5 \%$ thioflavin-T; the stained samples were viewed under UV light in a fluorescent microscope.

\subsection{Congo Red binding}

Samples were tested for amyloid-specific Congo Red binding by the spectroscopic band-shift assay described by Klunk [8]. Aliquots of $10 \mathrm{mg} / \mathrm{ml}$ protein were diluted in reaction solution $(5 \mathrm{mM}$ sodium phosphate/150 mM NaCl pH 7.0) containing $5 \mu \mathrm{M}$ Congo Red and absorption spectra were collected together with negative control solutions of dye in absence of protein and of protein samples in the absence of dye, subtracting the absorption of the dye and the scattering contribution from the samples spectra.

\subsection{Electron microscopy}

Protein samples at $10 \mathrm{mg} / \mathrm{ml}$ in buffer $\mathrm{F}$ were diluted 20 times in the same buffer and $5 \mu \mathrm{l}$ placed on a carbon-coated copper grid, and allowed to stand $2 \mathrm{~min}$. The grid was washed with distilled water and the sample stained with $2 \%$ uranyl acetate for another $2 \mathrm{~min}$. The samples were then evaluated in a Hitachi transmission electron microscope operating at an accelerating voltage of $75 \mathrm{kV}$.

\section{Results}

Formation of amyloid fibrils is often a slow process in competition with amorphous aggregation [2]. Several spectroscopic techniques can be used distinguish between $\beta$-sheet aggregation and fibril formation combined with direct observation of the fibrils by electron microscopy (EM). In many cases both 


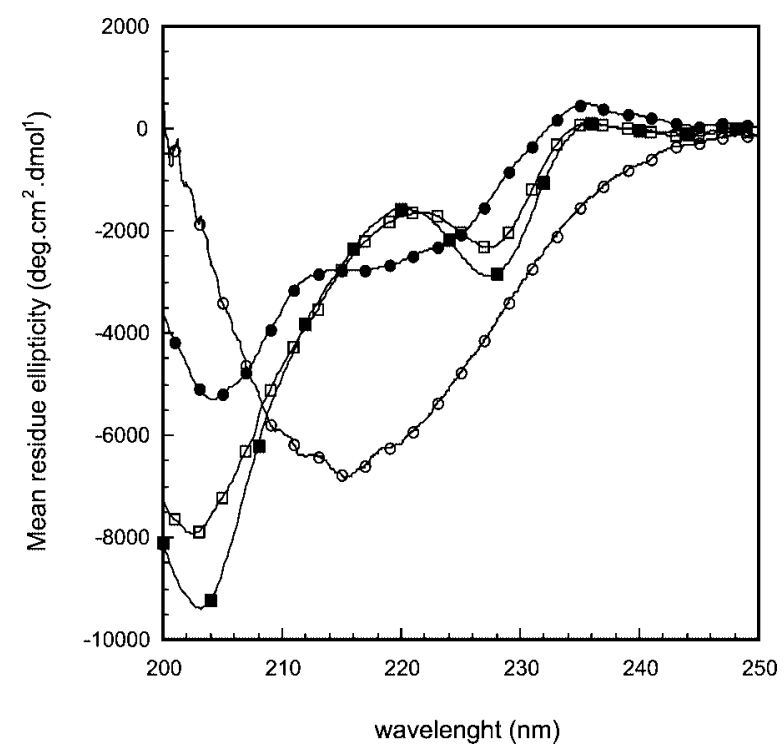

Fig. 1. CD spectra of SH3 domains in the far $\mathrm{UV}$ at $\mathrm{pH} 7.0$ (filled symbols) and $\mathrm{pH} 2.0$ (empty symbols) at $25^{\circ} \mathrm{C}$. $\mathrm{SPC}-\mathrm{SH} 3$ domain (squares) and PI3-SH3 (circles).

fibril and aggregation phenomena are slow and concentration dependent [9]. We used different spectroscopic approaches to follow the conformational changes over time of both PI3-SH3 and SPC-SH3 proteins and EM to find out if these changes were the result of aggregation or fibril formation.

When PI3-SH3 is incubated at $10 \mathrm{mg} / \mathrm{ml}$ concentration and $25^{\circ} \mathrm{C}$ under acidic conditions $(\mathrm{pH} 2.0)$ a translucent gel is formed in the lapse of hours. On the other hand, under the same conditions, SPCSH3 keeps in transparent solution even after several months. To monitor any conformational change responsible of this process we spectroscopically characterised the proteins at neutral $\mathrm{pH}(\mathrm{pH}$ 7.0), where both proteins remain in its native state, and under the acidic conditions that promote polymerization.

The CD spectra of both PI3-SPC and SPC-SH3 domains at pH 7.0 (native state) is very characteristic and mainly determined by the aromatic contribution of the triptophans that depends on tertiary [10] environment (Fig. 1).

After two weeks of incubation at acidic $\mathrm{pH}$ we acquired the far-UV CD spectra of the two proteins. PI3-SH3 displayed CD spectra typical of $\beta$ sheet structure as revealed by the single negative band at 215$220 \mathrm{~nm}$. The shape of the spectrum does not change with $\mathrm{pH}$ for SPC-SH3, even if the band intensity is lower, as expected if the protein is still folded and keeps soluble at pH 2.0 (Fig. 1).

Thioflavin- $T$ is an amyloid azo-free diagnostic dye that by an unknown mechanism specifically interacts with the crossed- $\beta$-pleated sheet structure common to a variety of amyloid fibrils. This property allows, in principle, the ThT assay to distinguish highly ordered amyloid structures from amorphous precipitates, which light scattering, a commonly employed method to evaluate amyloid formation, cannot. The binding of this dye to ordered, crossed $\beta$-sheet aggregates results in dramatic fluorescence emission [11-13]. Addition of thioflavin-T (Th-T) to PI3-SH3 and SPC-SH3 domains (Fig. 2), incubated for 15 days at acidic pH resulted in the following: PI3 increases by 5 -fold in the emission at $482 \mathrm{~nm}$. This change in fluorescence is consistent with PI3 having and amyloid structure, and was proportional to protein concentration on the assay. No apparent change in the emission spectrum was detected when thioflavin-T was added to the SPC-SH3 domain, in concordance with the CD spectra (Fig. 2). 


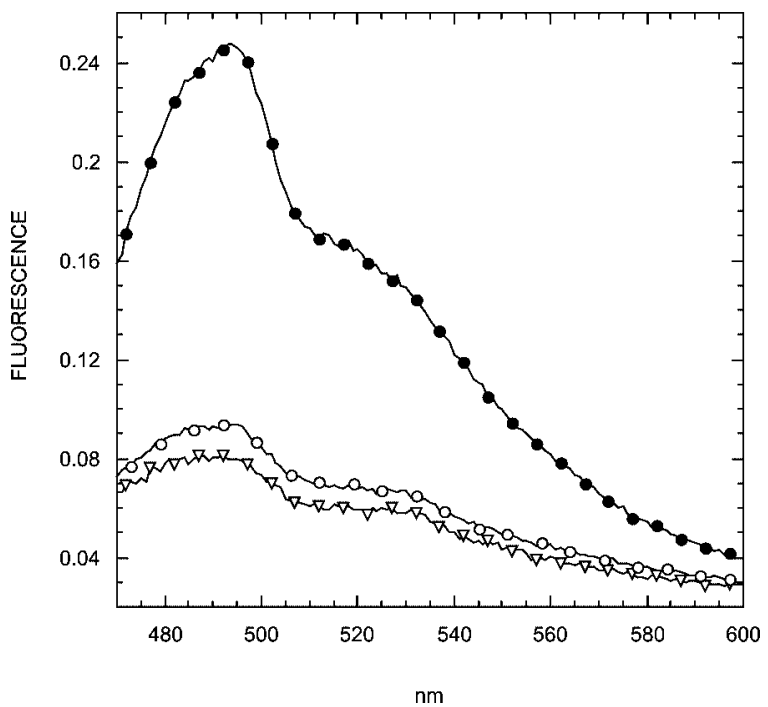

Fig. 2. Thioflavin-T binding. Fluorescence emission of thioflavin-T in the presence of SH3 domains incubated under acidic conditions for longer than two weeks. SPC-SH3 domain (empty circles), PI3-SH3 (filled circles) and thioflavin in the absence of protein (triangles).

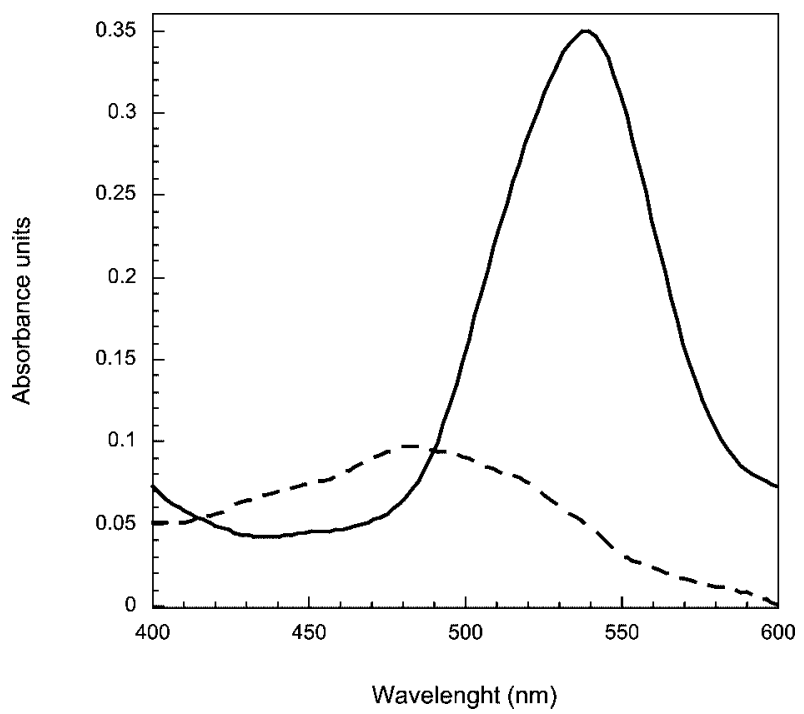

Fig. 3. Binding to Congo Red. Absorbance spectra of Congo Red in the presence of SH3 domains incubated under acidic conditions for longer than two weeks. SPC-SH3 domain (dashed line) and PI3-SH3 (solid line).

We further investigated the amyloid like properties of the SH3 domains by measuring the binding to another amyloid diagnostic dye [8], Congo Red, which also binds to all amyloids due to their betapleated fibrillar structure. Congo Red is suggested to bind to amyloid protein through a combination of both hydrophobic and electrostatic interactions. The need for specific interaction makes single-molecule Congo Red binding rather unusual as general amyloid protein ligand. The structural feature of Congo Red, which enables specific and common interaction with amyloid proteins, probably derives from the ribbon-like self-assembled form of the dye [14-17]. Congo Red alone exhibits an absorbance maximum 

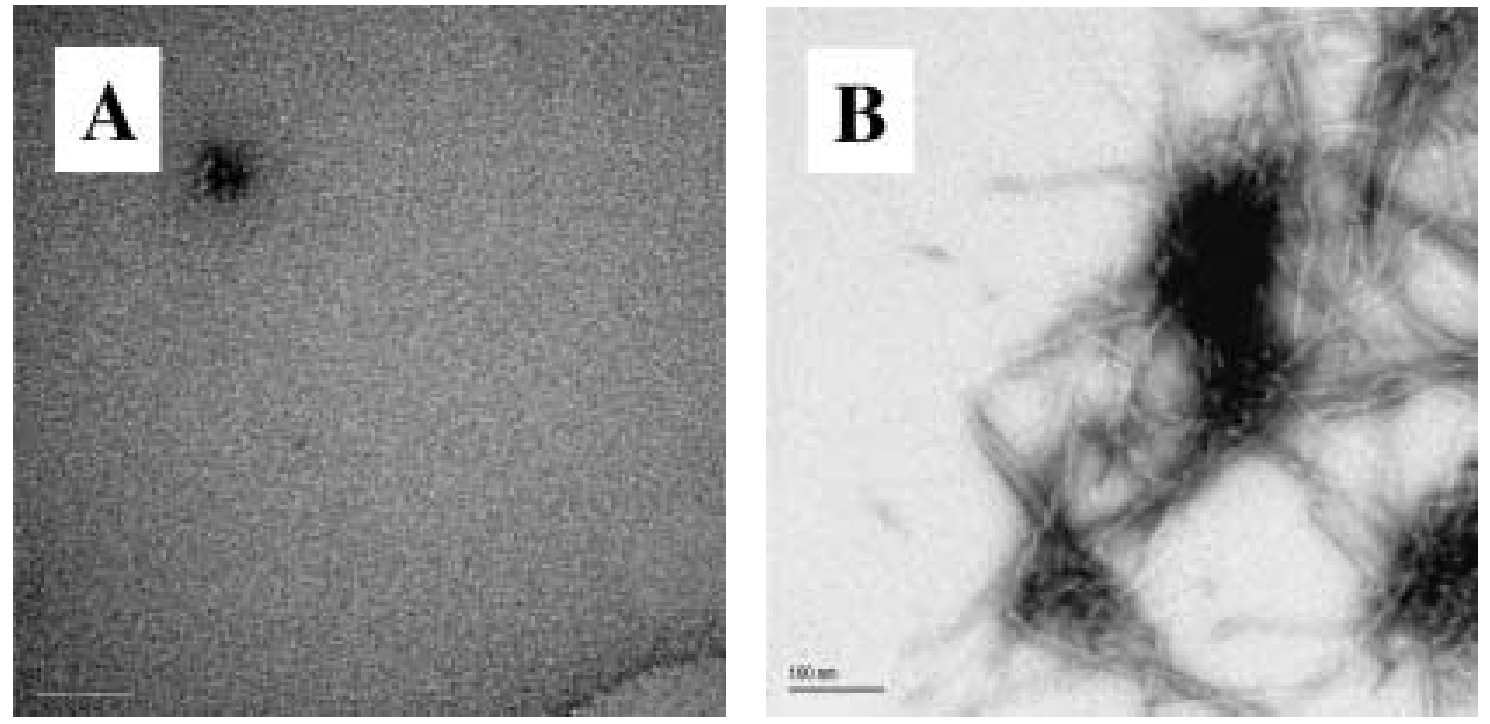

Fig. 4. Representative images of more than two weeks incubated SH3 protein solutions produced by transmission electron microscopy. The samples for electron microscopy were produced by a 20 -fold dilution of the originals samples used for incubation. (A) SPC-SH3 and (B) PI3-SH3 domains. The bar represents $1000 \AA$.

at $490 \mathrm{~nm}$ that increase and shifts to red once it binds to amyloid material [8]. PI3-SH3 promotes the expected changes in absorbance spectra while no variations on the spectra are recorded for SPC-SH3. The changes can be better quantified by obtaining the difference spectra between the dye alone and when incubated with the protein. After correction shows a maximum change at $540 \mathrm{~nm}$, similar to AB amyloid peptide [8] and other prion proteins while no such effect was observed for SPC-SH3 (Fig. 3).

While binding of both thioflavin-T and Congo Red to amyloid structures can be evaluated under the microscope due to the change of their fluorescence and emission properties, we preferred spectroscopic assay in solution, since microscopic techniques have several disadvantages that limit their utility in evaluating amyloidogenesis. Microscopic methods use samples that have been dried, introducing large increases in substrate concentrations during this process. Consequently sample preparation can contribute to the amount of observed amyloid, compromising the quantitative evaluation.

To test if the different spectroscopic properties of both molecules are really related to their aggregation conformation, solutions of proteins incubated for longer than two weeks at acidic $\mathrm{pH}$ were analysed by Electron Microscopy. Results are shown in Fig. 4. No depositions, or particles, were detected for spectrin SH3 domain in accordance with the above-mentioned results. Numerous fibrils displaying the typical amyloid morphology were observed in PI3-SH3 samples, showing that in the case of SH3-domains their spectroscopic properties are predictive of their conformation at acidic $\mathrm{pH}$.

\section{Conclusions}

Spectroscopic techniques have been used to test the fibril formation ability of two SH3 domains. Results obtained by spectroscopy are diagnostic and directly correlated for what is seen by direct observation using EM. By these criteria the SPC-SH3 domain form do not aggregate while PI3-SH3 forms ordered amyloid fibrils. The use of spectroscopic approaches in the study of amyloid diseases should help to find key structural/sequential features determinant for polymerisation. 


\section{Acknowledgements}

This work was financed by two European Union grants: Serrano/CT96-0013 and BIO4-CT97-2086.

\section{References}

[1] S.Y. Tan and M.B. Pepys, Amyloidosis, Histopathology 25 (1994), 403-414.

[2] M. Sunde and C.C. Blake, From the globular to the fibrous state: protein structure and structural conversion in amyloid formation, Quart. Rev. Biophys. 31 (1998), 1-39.

[3] C.M. Dobson, The structural basis of protein folding and its links with human disease, Phil. Trans. Roy. Soc. London 356 (2001), 133-146.

[4] J.I. Guijarro, M. Sunde, J.A. Jones, I.D. Campbell and C.M. Dobson, Amyloid fibril formation by an SH3 domain, Proc. Natl. Acad. Sci. USA 95 (1998), 4224-4228.

[5] C.M. Dobson, Protein misfolding, evolution and disease, Trends Biochem. Sci. 24 (1999), 329-332.

[6] SH3 domains: complexity in moderation, J. Cell Sci. 114(Pt 7) (2001), 1253-1263.

[7] S. Ventura, E. Lacroix and L. Serrano, Insights into the origin of the tendency of the PI3-SH3 domain to form amyloid fibrils, J. Mol. Biol. 322 (2002), 1147-1158.

[8] W.E. Klunk, J.W. Pettegrew and D.J. Abraham, Quantitative evaluation of congo red binding to amyloid-like proteins with a beta-pleated sheet conformation, J. Histochem. Cytochem. 37(8) (1989), 1273-1281.

[9] J. Zurdo, J.I. Guijarro, J.L. Jimenez, H.R. Saibil and C.M. Dobson, Dependence on solution conditions of aggregation and amyloid formation by an SH3 domain, J. Mol. Biol. 311(2) (2001), 325-340.

[10] A.R. Viguera, J.C. Martinez, V.V. Filimonov, P.L. Mateo and L. Serrano, Thermodynamic and kinetic analysis of the SH3 domain of spectrin shows a two-state folding transition, Biochemistry 33(8) (1994), 2142-2150.

[11] H. LeVine, 3rd, Thioflavine T interaction with synthetic Alzheimer's disease beta-amyloid peptides: detection of amyloid aggregation in solution, Protein Sci. 2(3) (1993), 404-410.

[12] H. LeVine, 3rd, Stopped-flow kinetics reveal multiple phases of thioflavin T binding to Alzheimer beta (1-40) amyloid fibrils, Arch. Biochem. Biophys. 342(2) (1997), 306-316.

[13] C. Goldsbury, K. Goldie, J. Pellaud, J. Seelig, P. Frey, S.A. Muller, J. Kistler, G.J. Cooper and U. Aebi, Amyloid fibril formation from full-length and fragments of amylin, J. Struct. Biol. 130(2-3) (2000), 352-362.

[14] A. Lim, A.M. Makhov, J. Bond, H. Inouye, L.H. Connors, J.D. Griffith, B.W. Erickson, D.A. Kirschner and C.E. Costello, Betabellins 15D and 16D, de novo designed beta-sandwich proteins that have amyloidogenic properties, J. Struct. Biol. 130(2-3) (2000), 363-370.

[15] I. Roterman, M. KrUl, M. Nowak, L. Konieczny, J. Rybarska, B. Stopa, B. Piekarska and G. Zemanek, Why Congo Red binding is specific for amyloid proteins - model studies and a computer analysis approach, Med. Sci. Monit. 7(4) (2001), 771-784.

[16] B. Piekarska, J. Rybarska, B. Stopa, G. Zemanek, M. Krol, I. Roterman and L. Konieczny, Supramolecularity creates nonstandard protein ligands, Acta Biochim. Pol. 46(4) (1999), 841-851.

[17] W.G. Turnell and J.T. Finch, Binding of the dye congo red to the amyloid protein pig insulin reveals a novel homology amongst amyloid-forming peptide sequences, J. Mol. Biol. 227(4) (1992), 1205-1223. 


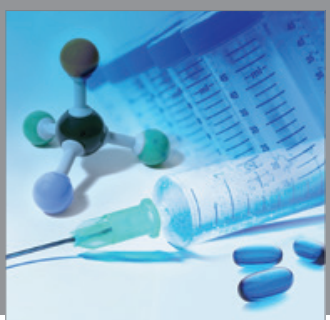

International Journal of

Medicinal Chemistry

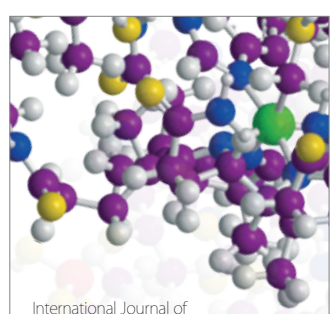

Carbohydrate Chemistry

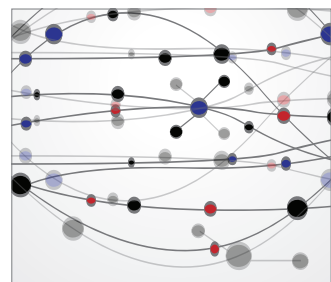

The Scientific World Journal
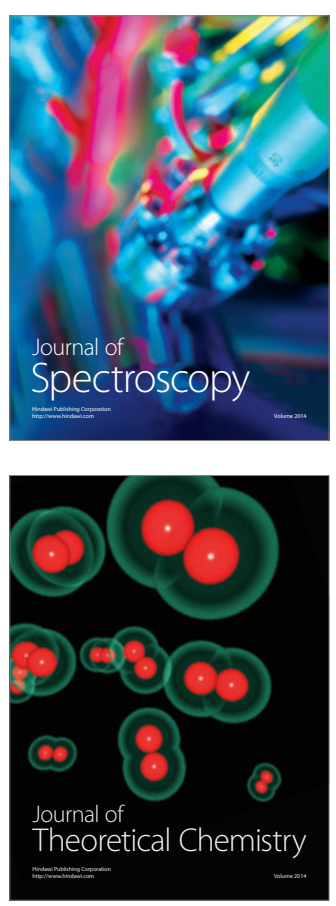
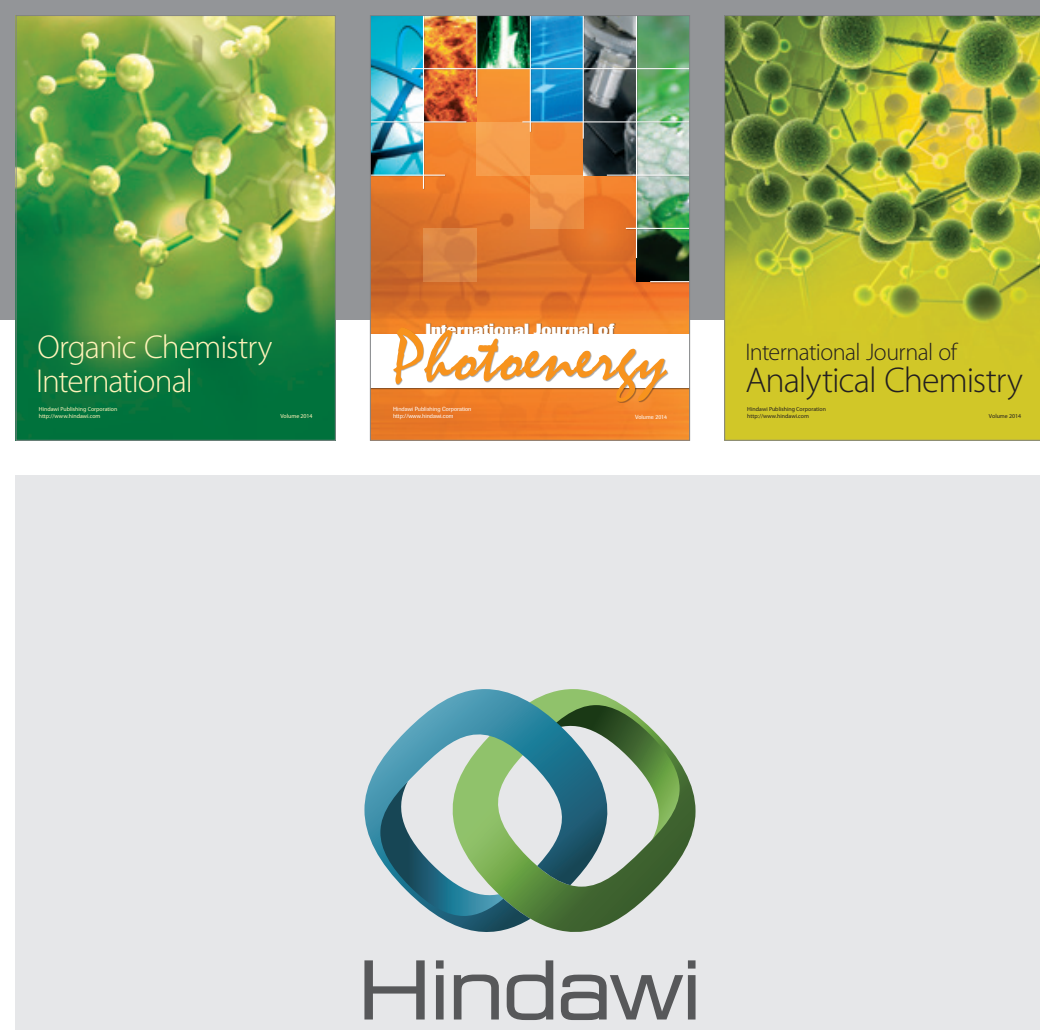

Submit your manuscripts at

http://www.hindawi.com
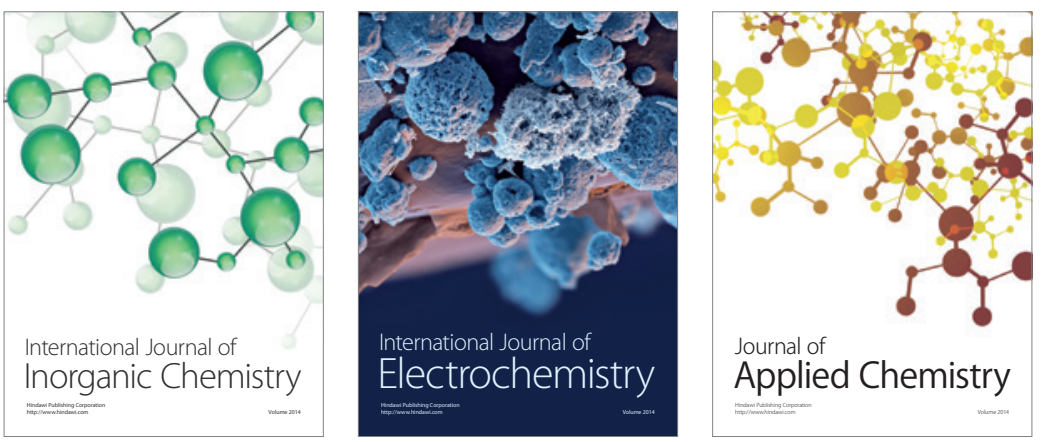

Journal of

Applied Chemistry
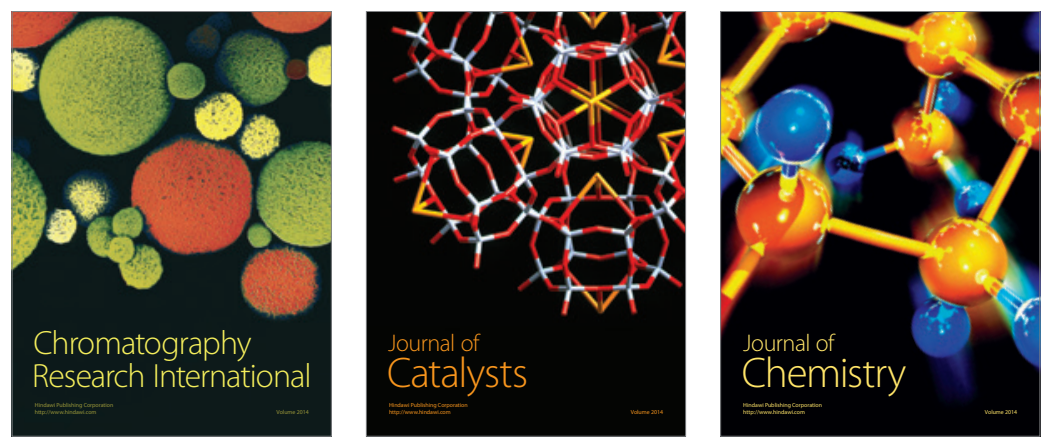
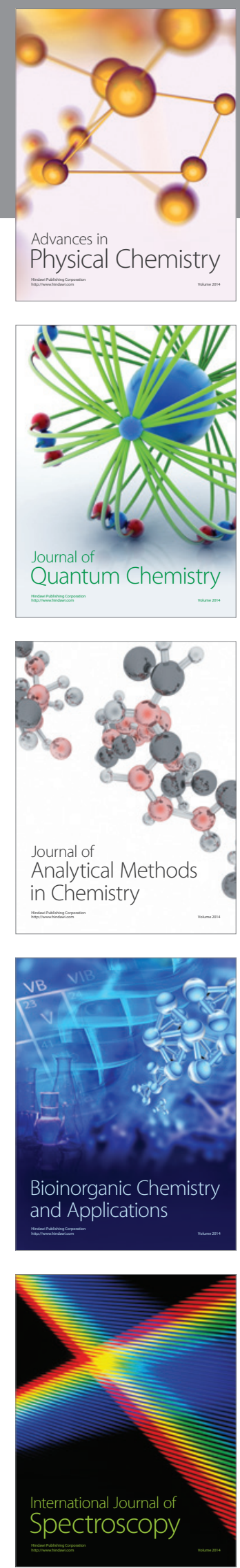